\title{
A QoS towards Dynamic Web Services Recapitulation and Selection
}

\author{
Ashok Kumar.P.S \\ Research Scholar, \\ Anna University, Coimbatore,
}

\author{
G. Mahadevan, Ph.D \\ Prof \& Head \\ AMCE, Bengaluru
}

\author{
Gopal Krishna.C \\ Asst. Prof, Dept. of CSE \\ AIT, Chikmagaluru
}

\begin{abstract}
Web services are the components, communicates via Internet or Web. Fundamental vision of the Web service is a brokerage system. The Recapitulation of Web service is an approach for the effective integration of distributed, heterogeneous and autonomous applications to build more Structured and value added services. Web services selection algorithms are required to find and select the best services. A QoS is a benchmark to select the best service for the task of composition. The QoS broker based web service selection plays an important role in service-oriented computing, where most of the applications are user specific QoS services. Here we propose a Broker based architecture for selection of web services. The importance of the web services selection algorithm is to maximize the QoS of the web services recapitulation using complex service provider's (CSP) QoS requirement parameters. This paper have discussed about different Web service Service-offers. We have classified the CSP's requirements defined on the QoS and Service-offers based on its structure. This paper presents an efficient method to finding the suitable web service based on the consumer's requirements along with QoS.
\end{abstract}

\section{General Terms}

Algorithm, Recapitulation, web service

\section{Keywords}

Recapitulation, Service selection, QoS, SOA, UDDI, tModel

\section{INTRODUCTION}

A Web service is an API that describes a collection of operations that are accessible through Internet based on standardized XML messaging. Web services can be published, found and used across the Internet using SOAP, WSDL and UDDI standards. Web Services are encapsulating application functionality and information resources. The main emphasis of Web services is to save development time and cost through the reuse of components [1] [2]. The Web service architecture is based on the interactions between service requester,
Service registry and service provider, where the interactions between the services involve publish finding and bind operations [1].

If multiple Web services provide the same functionality, then a Quality of Service (QoS) requirement can be used as selection services. QoS is a set of attributes, like reliability service response time, throughput and availability [3]. Or QoS (Quality of Service) is a combination of Web service qualities, and it evaluate of how well the Web service convey the information to requester.

The QoS of web services requirements are mainly intends to the quality aspect of a web service. These may include performance, reliability, scalability, capacity, robustness, exception handling, accuracy, integrity, accessibility, availability, interoperability, security. A Technical Model (tModel) is a data structure, it represents an XML Web services type in a Universal Description, Discovery and Integration (UDDI) registry or t-Model represents a generic representation of a registered service in a UDDI registry [17]. The tModel provide generalization for a technical specification of a service type, i.e. tModel organizes the information of a particular service type and makes it accessible in the registry database [15]. Web services are loosely coupled in nature. The travel agent doesn't need to have prior agreements with service providers or credit card companies [3]. This allows the travel agent to access more services, offer more options to their customers, i.e. the credit card companies offer their services broadly to make their customers happy, as the service providers offer their services broadly and easily they are generating more business for themselves. QoS can be used for selection and ranking of the Web services by extending standard service oriented architecture (SOA).

In this paper, we have defined various Service-offers and proposed a tree structure to represent the CSP's requirements limited in the QoS and Service-offers [11]. 


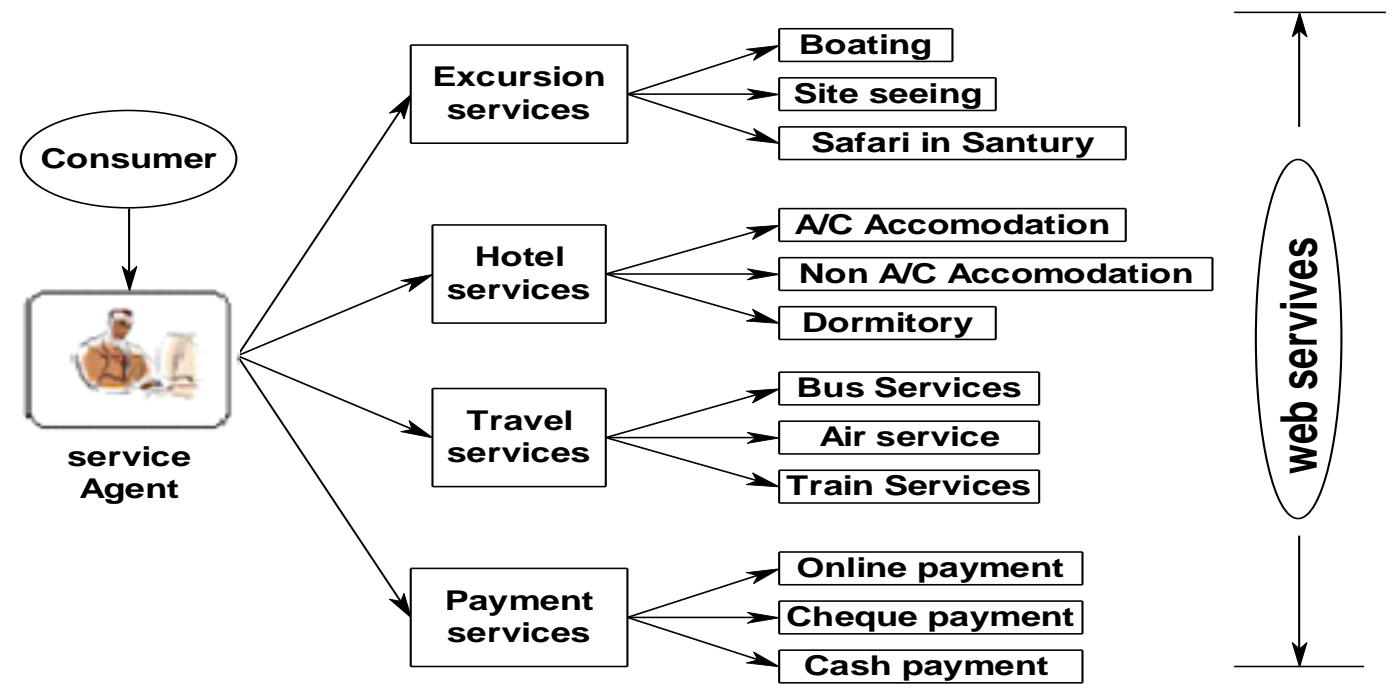

Fig 1: Travel Reservation Application

\section{Case study 1:}

Travel Reservation Application -

The travelers normally prefer reservation for his/her distant travel location through travel agent. The main objective of a traveler is to get the best combination of services like, quality, price and valid offers which satisfy his/her needs. On the other hand, travel agent tries to satisfy the customer's needs and mint money by charging extra fees like service charge for each trip.

In this Travel Reservation Application, travel agent is a service Intermediator, who has to find the best services for the individual tour package based on the traveler's demands on the service quality and offers. Figure 1 shows the Travel Reservation Application. The travel agent uses the Web service system to find and integrate different services that are provided by the different travel service providers. The travel agent service can publish the specific service information into service registry, but QoS of the service is permitted to create a document either in digital or hard copies, in whole or part of a traveler.

To republish, or to post on servers, agent requires prior specific permission or a traveler has to pay specified trip fee, depending on the QoS of constituent services. With respect to the above mentioned example, the customer's Travel Reservation application consists following activities:

(a) Book an Air ticket from Bangalore to Mauritius.

(b) Book a single AC Room in Mauritius star Hotel for 02 days.

(c) Book a Taxi in Mauritius from airport to Hotel.

(d) Book a ticket for Mauritius city tour.

(e) Booking for the dinner at Mauritius Beach Hotel.

The customer's QoS and business offer requirements are as follows -

* The price should be minimum.

* The most esteemed service offers with good discount.

When travel agents get such requirements from a traveler, the agent has to find the service that satisfies all requirements of a traveler. Usually a Travel agent is interested in reliable travel service provider to improve the reliability of a travel composite service [1] [13].
The Composite or Complex service provider (CSP) defines the requirements to travel agents on the multiple QoS properties and Service-offers involving AND/OR operators, it is very tedious work to find the best Web services for the individual task of the recapitulation. This paper addresses few issues related to service selection in recapitulation. This paper is organized as;

Section 2 specifies QoS Broker Architecture, Section 3 defines the Web Services Recapitulation Requirements, Section 4 defines the QoS Selection Algorithm and Section 5 depicts the conclusion and future work.

\section{QoS BROKER ARCHITECTURE}

Quality of Service (QoS) is a combination of several qualities or attributes of a service. QoS is a collection of nonfunctional attributes that may affect the quality of the service provided by a Web service [3] [8].

\subsection{QoS Model}

In order to attract the customers, the service providers normally advertise a lot, as well as display with attractive offers to improve their business with huge profit. In this section, we define Service-offer vocabulary from the requester's point of view. This paper defines the Service-offers as a reduction in the service price or providing the services as they advertise or offering an article as a gift for the service consumption, etc... [1][2][16]. This paper broadly categorizes various web services Service-offers

QoS model of Web service includes the Service-offers with in the business specific QoS category. Figure 2 shows the major QOS model of Business Specific Offer categories. The service provider can advertise multiple offers which may belong to the same kind or different kind [8] [14]. For example, the travel reservation service provider may advertise offers like, 'confirm four reservations, one reservation and one lucky coupon is free', these Service-offers are recorded in a separate Service-offer table. Usually the Service-offer table contains mandatory information like, Types of Service-offer, information of the offer and service-keywords of the specific advertised Service-offers. 


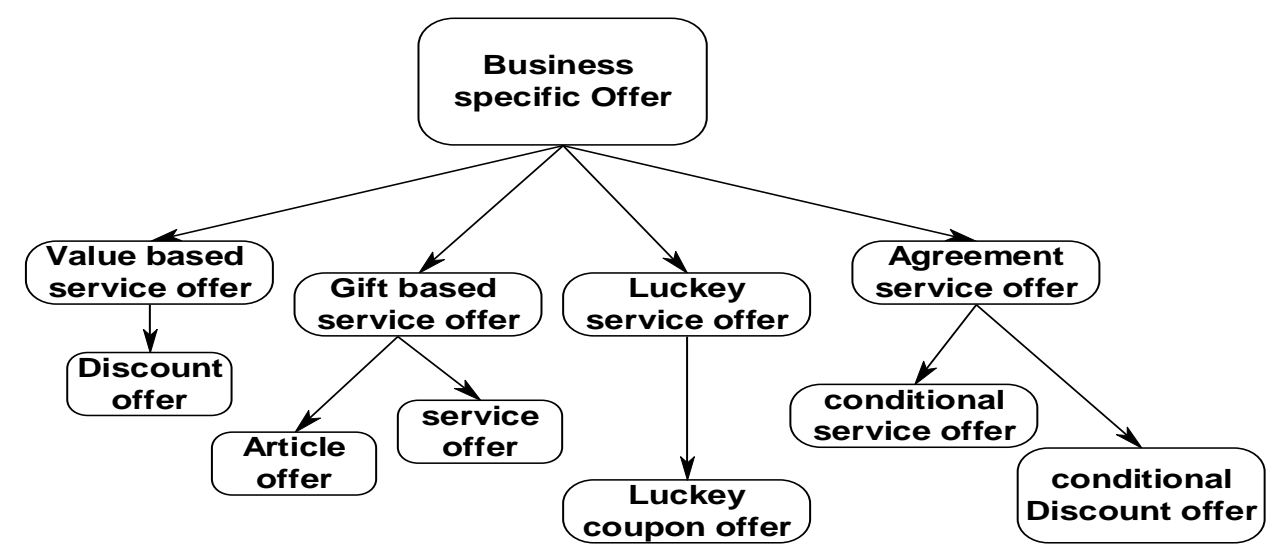

Fig 2: QOS model of Business Specific Offer

The QoS is most important for Web service publishing and selection of service activities. The parameters in a Service-offer are, price is a real number which indicates the currency, percentage refers to a number in the range, period refers to time period in hours or days and frequency refers to number of service executions (always greater than one). The Service-offer listings which are defined in this model have to be used by all Web service providers and service requesters for the Serviceoffer [3].

The architecture consists of basic web services components, like service provider, service consumer, QoS Broker and QoS registry. QoS registry has the ability to store all QoS information based on tModel. Web Services QoS Broker component consists of Web Service Registry, QoS manager, QoS Verifier \& Certifier, and QoS Registry. The QoS Broker assists clients in selecting web services based on a set of QoS parameters. QoS Broker services may be used to facilitate service registry access based on the interaction with the UDDI registry [3] [13] [19].
The main components of Qos Broker Architecture are -

\section{i. Service Selector}

The service selector component is referred to select the most desired web service to satisfy the consumer's QoS requirement as well as specific service. It receives messages from the web service consumer, specifying the service functionality along with the QoS requirements.

\section{ii. Service Publisher}

The service publisher component conveys the information to the service provider and the UDDI registry. The web service provider registers the web service through the service publisher. i.e. The Service publisher is gets the specific QoS property values from service provider.

\section{iii. Verifier}

This is the key component of the QoS Broker architecture, it performs the verification of the QoS requirement information supplied by the service provider and issues a certificate to the service provider through the service publisher.

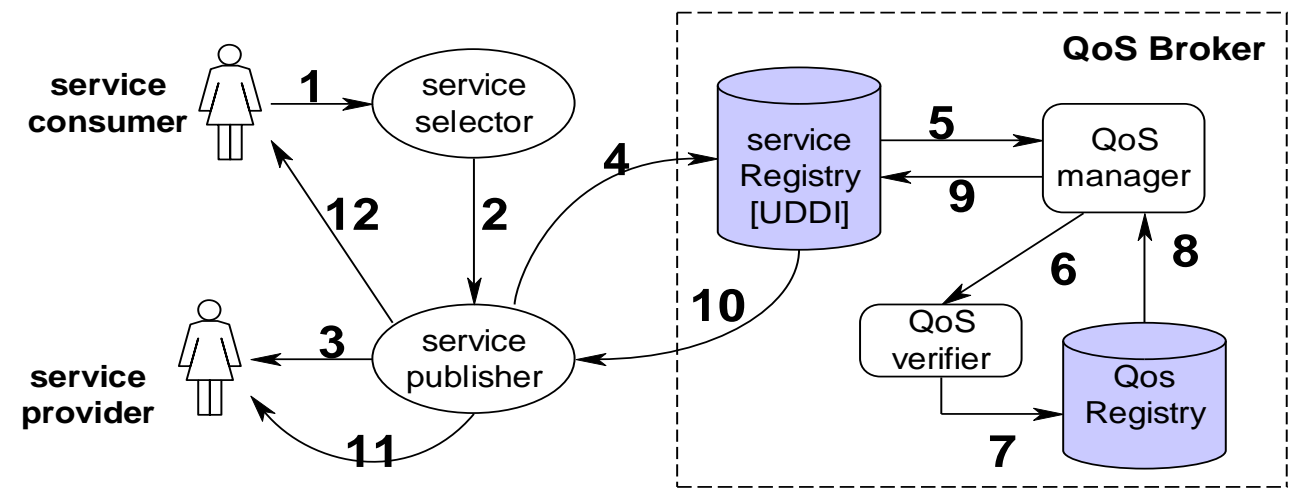

1. Service request

2. Select \& publish service

3. Request to provider

4. Verify in registry

5. Request for Qos

6. Verify the QoS
7. Find QoS in registry

8. Select QoS requirement

9. Get Qos requirement

10. Publish Qos service

11. Information to service provider

12. Service response

Fig 3: QoS Broker based Architecture 
The web services QoS broker is also a web service, it enables the architecture deployment in an open environment. Figure 3, depicts web services QoS broker based architecture, where the service publisher component facilitates the registration, updating and deletion of web service related information. It gets the business specific and performance specific QoS property values of web services from the service providers. The service provider publishes its service function to the UDDI registry through the service publisher [17]. The service publisher will complete the specific certification and verification based on $\mathrm{QoS}$ requirement of the service consumer. Figure 4, shows the sequence diagram for Broker based Architecture, it shows how the customer will get quality web services.

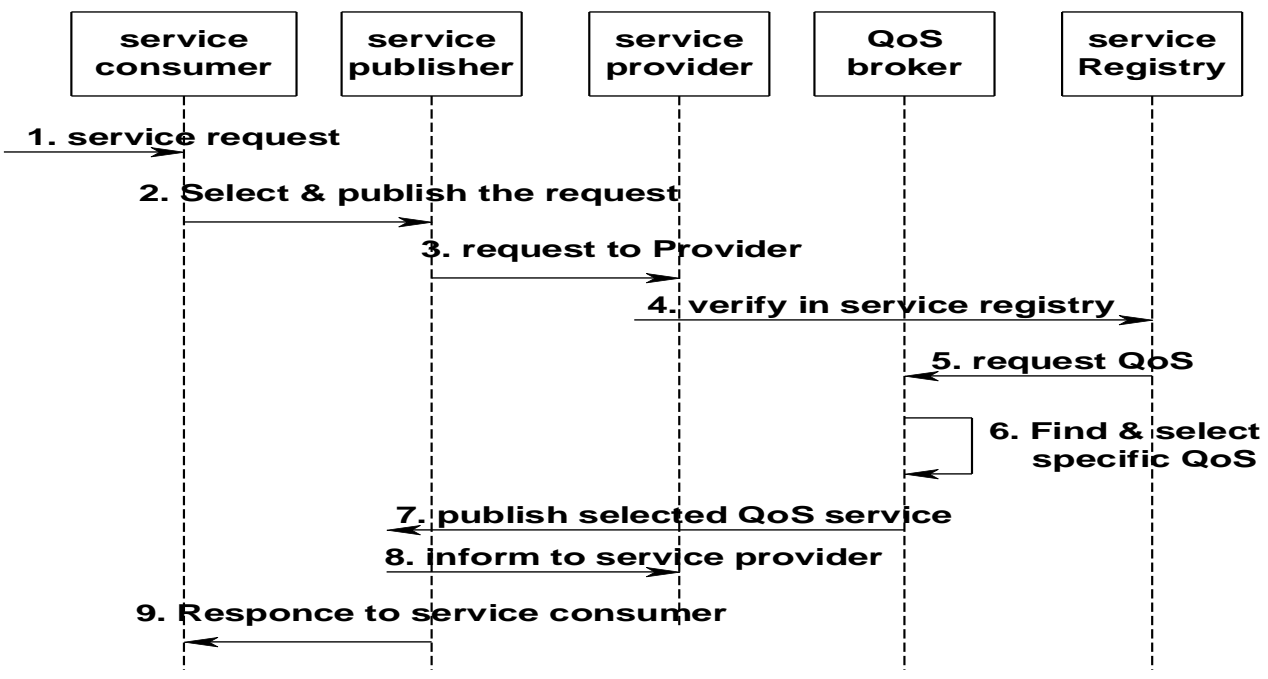

Fig 4: Sequence diagram for Broker based Architecture

For every service a service publisher handles all communication with service registry, binding, selection, request and responses for that specific service. i.e. the service consumer can search the UDDI registry for a specific service through the service selector. The importance of the service selector component is to select the most desired web service satisfying requester's QoS requirement, along with service functionality [21]. The QoS broker performs the verification and certification of service request from service consumer. QoS verification is the process of validating the information described in the service interface as well as described QoS parameters [5] [6].

The QoS information is specified in the UDDI registry by using a tModel, it contains specification, standardization and reuse of QoS information. i.e. tModel allows the use of QoS broker to facilitate service selection based on functional and non-functional requirements and verify QoS characteristics[15].

Some QoS parameters are -

i. Availability is the probability of the system is available at every moment.

ii. Reliability is the ability of a service to perform its required functions under specified condition within a specific period of time.

iii. Cost is the price for requesting a specified service.

iv. Performance is the evaluation time to complete a service request. It is measured by latency, throughput and response time.

v. ResponseTime is the Time taken by a service to respond to the client request.

vi. Throughput is the maximum requests that can be handled at a given unit of time.

\section{2 tModel and its Taxonomy}

tModel is a generalization for a technical specification of a service type, it organizes the service type information and makes it accessible in the registry database. tModel are used in UDDI registry to represent unique idea. tModel provide a structure that enables reuse of services based on shared requirements within architecture [15].

A tModel is used to represent taxonomy or hierarchical system, which allows service provider to be classified depending on the nature of their business activities, or A tModel, is simply a classification system with a finite set of values [5]. Figure 5. represents a tModel with QoS requirement information.

For instance, A Travel Service System, Each taxonomy system is represented by a tModel. A tModel consists of a key, a name, an optional description and a Uniform Resource Locator (URL), that point to the location where the details about the actual concept can be found. When a service is published in the UDDI registry, a tModel is created to represent the QoS information of the service. It is registered with the UDDI registry and referenced in the binding Template that represents the deployment information of the web service [17].

$<$ tModel tModelKey $=$

"TravelService:QoSInformation">

$<$ name>QoS Information for Travel Service</name> $<$ URL http://< describing the schema for QoS attributes $>$

$\langle/$ URL $>$

$<$ level $>$ 


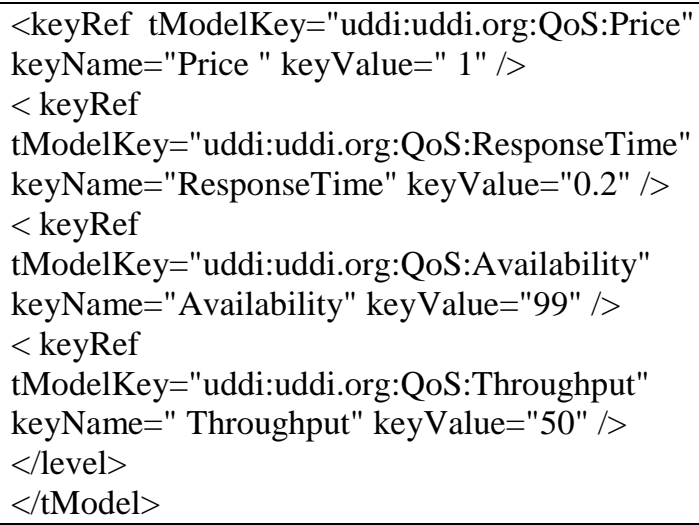

Fig 5: tModel with QoS for Travel Service Application

\section{WEB SERVICES RECAPITULATION REQUIREMENTS}

Recapitulation requirements are the requirements which are defined based on the QoS and Service-offered by the CSP. The recapitulation requirements different for each and every service providers, which are dependent on the customers or requester's basic needs or service provider's competition of service or target in achieving the profit[1]. The CSP also gives preference or weight age to each service requirement. For example, the CSP may compose the services, which are low cost and fast response or low cost with high reputation, security and discount offer etc. consider the travel package scenario; here the requirements on the service price and service offers that are necessary to execute the complex service in a profitable manner. Thus CSP can impose different requirements on several QoS properties and/or Service-offers with varied preferences. For example, a Recapitulation requirement of a customer is requested for a Service-offer to choose a Flight as well as Hotel Accommodation in Malaysia, through service Agent. The recapitulation requirements service offer steps are given below,

1. The travel agent service finds a list of airlines.

2. The customer communicates his/her choice for the flight.

3. The airline returns a confirmation number with an expiry date.

4. The travel agent service requests the chosen airline to put the flight on hold:

i. The travel agent service requests a description of how to put a seat on hold to the airline service.

ii. The travel agent service sends the request based on customer's priority.

5. Identification of Accommodation in the Hotel by Travel Agent.

i. The travel agent communicates with the Hotel manager for the description of service found

ii. He requests the accommodation options for the period.

6. The travel agent service looks for the payment services available, and builds a list of options for the customer.

7. The travel agent service delivers the results of the queries to the customer to choose their best option, along with the payment options that offered.

\subsection{Recapitulation Requirement Types}

Recapitulation requirements are different for service providers and service requester, for their Reputation and security. we classify complex service provider's (CSP) requirements based on the requirement structure as;

$$
\text { * Complex requirements. }
$$

A simple requirement is defined based on a single QoS property or a single Service-offer. For example, the CSP might say, 'they provide minimum cost of the Hotel Accommodation service '. If the simple requirement is defined on the QoS property, then it is called as a Simple Quality Requirement.

A complex requirement is composed of two or more simple requirements using recapitulation operators AND \& OR. For example, the CSP might say, 'they provide minimum cost and more discount in their services '. The CSP can enforce either a simple or complex requirement with varied preferences for the selection to build a desired quality complex service.

\subsection{Recapitulation Requirement Modeling}

The recapitulation requirements are to be fulfilled based on the QoS properties and/or Service-offers with reference to the simple and/or complex web service requirements [3]. In this paper, we propose a tree structure called Recapitulation Requirement Tree, which represents the complex service provider's (CSP) recapitulation requirements.

\subsubsection{Recapitulation Requirement Tree (RRT)}

A recapitulation requirement tree is a AND-OR tree, with Weighted node [1] whose leaf node contains the QoS property or Service-offer. For Example: if we consider the travel agent's requirements in order to increase the profit in the travel package booking, he has to follow:
(1) Discount offer (DO)
(2) Service offer (SO)
(3) Popular offer (PO)
(4) Lucky coupon offer (LCO)

The CSP expects both the requirements to be equally satisfied for the recapitulation with preferred weights 0.4 , 0.6, 0.7 and 0.3 to Discount offer, Service offer, and Lucky coupon offer respectively. This recapitulation requirement can be represented as the RRT in Figure 6 .

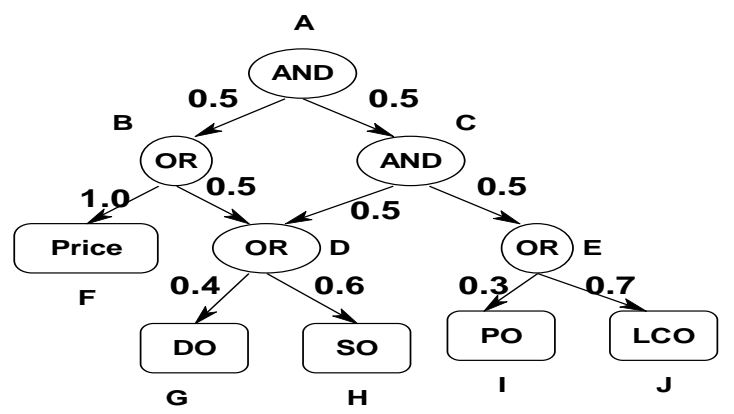

Fig 6: Recapitulation Requirement Tree 
The node $\mathrm{J}$ represents the simple offer requirement and the node $\mathrm{H}$ represents the simple quality requirement. The subtree at node $\mathrm{D}$ represents the complex requirement and the weight $(0.5)$ on the edge $(\mathrm{B}, \mathrm{C})$ represent the preference for the complex requirement defined at node B.

\subsection{QoS SELECTION ALGORITHM}

A web service consumer sends a service selection request to the service selector, which communicates with UDDI registry to find services that meet the customer's functional as well as QoS requirements. If a service is said to be a "match" or "equal", i.e. the service is satisfies the customer's requirement and QoS constraints. If a service is "not-match", then the service selector returns an empty result to the customer like "service is Not Matched". If multiple services match the functional and QoS requirements, the service selector calculates a QoS values for each matched service based on the priority QoS attribute value specified by the customer, or on the default dominant attribute, average response time. For example, the best service is assigned a score of 1 , or keyValue $=" 1$ " and the other services are assigned scores based on the value of the priority QoS attribute.

\subsection{Web services Selection Mechanism}

In Web services recapitulation, the selection mechanism accepts the Recapitulation Requirement Tree (RRT) and requester Web services as an input and assigns the best Web service for that particular task. Here Web services Selection mechanism in RRT traverses in a bottom-up approach [1].

At the bottom most leaf nodes the selection mechanism performs two actions, like:
(1) Scaling

(2) Ranking.

In the scaling phase, the Web service selects a simple offer requirement. In simple offer requirement the price $(\mathrm{P})$ of a Web service is calculated. The P/QoS values of the selected Web services are normalized using min-max normalization technique [2].

In ranking phase, the normalized values are multiplied with the weight to get new scores for Web services [3]. At the internal node, the selection mechanism performs two actions:

\section{(1) Filtering \\ (2) Ranking}

Filtering \& Ranking selection mechanism are dependent on the types of internal node AND/OR operations.

In filtering phase, if the node is $A N D$, then the Web services present in entire child nodes are get selected.

If the node is $O R$ then the distinct Web services in the descending order of their scores are selected from its child nodes.

In ranking phase, if the node is $A N D$, then the score of selected Web service is computed. When the sum of the scores of selected Web service at its child nodes are multiplied, it results with the weight of sub-tree rooted at AND node [4] [11].
If the node is $O R$ then the score of selected Web service is multiplied with the weight of sub-tree rooted at $O R$ node. After ranking the Web services at the root node, they are sorted in the descending order of their scores, and the first Web service becomes the best choice for the task satisfying the CSP's recapitulation requirements.

\subsection{Algorithm for Web Service Matching, Ranking and Selection}

Figure 8, shows the algorithm for service matching, ranking and selection which is to be implemented by Web Service QoS Broker.

i. functionMatch1 returns a set of services meeting the functional requirements.

ii. qosMatch1 returns the services that meet the QoS requirements.

iii. ratingMatch returns the set services whose ratings are equal and above than rating requirements, from those returned by method qosMatch and sort them in descending order.

iv. qosRank computes the QoS ranks for those services returned by method qosMatch and sort them in descending order of QoS rank.

v. selectService returns a set of services depending upon the maximum number of services to be returned in response to the discovery request.

\section{$/ *$ Algorithm for web service matching, ranking and selection $* /$}

find_Services(fun_Req, qos_Req, rating_Req, max_Services)

$\{$ //find services for the functional requirements functionMatch1 = functionMatch(fun_Req);

if $\mathrm{QoS}$ requirements are specified

qosMatch1 = qosMatch(functionMatch1, qos_Req); else

return selectService(functionMatch1, max_Services, "byRandom");

if Rating requirements are specified

Match1 = ratingMatch(qosMatch1, qos_Req,

rating_Req);

return selectService(Match1, max_Services, "byQoS\&Rating");

else

Match1 = qosRank(qosMatch1, qos_Req);

return selectService(qosMatch1, max_Services, "byQoS");

Fig 8: Algorithm for Service Matching, Ranking and Selection

\section{CONCLUSION}

In Business offering Service-offers are most important for Web service selection. This paper intends a tree structure called Recapitulation Requirement Tree (RRT) to represent the multiple QoS properties and Service-offers involving AND/OR operators with varied preferences. QoS is an effective factor to identify dynamic web services selection and repetitive functional Web services. We proposed a novel approach to identify QoS based service selection. This approach reduces the complexity of 
matching the user request based on the specified functional and QoS requirements. The Qos broker Architecture performs the process of publishing and selection of web services. QoS broker based architecture will be based on QoS attributes. Based on QoS confined complex service selection algorithms, we can discover the near-optimal complex web services efficiently. In our future work, we plan to add a novel trust evaluation approach that aggregates the ratings from other customers or requesting client's to the service agents prior subjective belief about the trusted service.

\section{REFERENCES}

[1] Ashok kumar.P.S, G.Mahadevan, C.Gopal Krishna, Recapitulation of Web services based on Tree structure, International Journal of Computer Application, 2012,Vol. ACCTHPCA,Issue-1, pp. 3236

[2] Demian Antony D'Mello, V.S. Ananthanarayana and Santhi Thilagam, "A QoS Broker Based Architecture for Dynamic Web Service Selection", In Proceedings of the Second Asia International Conference on Modeling and Simulation (AMS 2008), May 13-15, 2008, Kuala Lumpur, Malaysia, IEEE Computer Society, pp. 101- 106, 2008.

[3] Demian Antony D' Mello, Santhi.T and V.S.Ananthanarayana, 2008, "A QoS Broker Based Architecture for Web Service Selection", Proceedings of IEEE International Conference.

[4] Kreger, "Web Services Conceptual Architecture (WSCA 1.0)", IBM Corporation Specification2001,Available:ww.ibm.com/software/s olutions/web services/pdf/wsca.pdf

[5] Menasce D. A., "Composing Web Services: A QoS View", IEEE Internet Computing, 2004. pp. 88-90.

[6] L. Yang, Y. Dai, B. Zhang and Y. Gao, "A Dynamic Web Service Composite Platform Based on QoS of Services", In Proceedings of APWeb Workshops 2006, LNCS 3842, pp. 709-716, 2006.

[7] T. Yu, Y. Zhang and K. Lin, "Efficient Algorithms for Web Services Selection with End-to-End QoS Constraints", ACM Transactions on the Web, Vol. 1, No. 1, Article 6, pp. 1-26, May 2007.

[8] Demian Antony D'Mello and V.S. Ananthanarayana, "A QoS Model and Selection Mechanism for QoSawareWeb Services ", In Proceedings of the International Conference on Data Management (ICDM 2008), Feb 25-27, 2008, Delhi, pp. 611621.

[9] Lei Li, Yan Wang, Ee-Peng Lim "Trust-Oriented Composite Service Selection with QoS Constraints “, Journal of Universal Computer Science, vol. 16, no. 13 (2010), 1720-1744
[10] Netra Patil \& Arpita Gopal, "Ranking Web-services Based on QoS for Best-fit Search “,International Journal of Computer Science \& Communication Vol. 1, No. 2, July-December 2010, pp. 345-349

[11] Demian Antony D'Mello, V.S. Ananthanarayana and Santhi Thilagam, " Semantic Web Service Selection Based on Business Offering ", In Proceedings of the Second Asia International Conference on Modeling and Simulation, May 13- 15,2008, Kuala Lumpur, Malaysia, IEEE Computer Society, pp. 476-481, 2008.

[12] Dongsong Zhang, Minder Chen, and Lina Zhou, "Dynamic And Personalized Web Services Composition In E- Business “, Information Systems management JOURNAL, 2005, pp. 50-65.

[13] Rajendran, T. and Balasubramanie, P., 2009, "An Agent- Based Dynamic Web Service Discovery Framework with QoS Support", International J. of Engg. Research \& Indu.Appls. (IJERIA), Vol.2(5),pp 1-13.

[14] Rajendran Thangavel and Balasubramanie Palanisamy “Efficient Approach Towards an AgentBased Dynamic Web Service Discovery Framework with QoS Support", 2009 International Symposium on Computing, Communication, and Control, Proc of CSIT vol.1 IACSIT Press, Singapore, pp 74-78

[15] tModel, http://msdn.microsoft.com/enus/library/ aa303717.aspx

[16] W3C, "QoS for Web Services: Requirements and Possible Approaches", Available: http://www.w3c.or.kr/kroffice/ R/2003/NOTE-wsqos-20031125/, 2003.

[17] Blum, A., 2004, "UDDI as an Extended Web Services Registry: Versioning, quality of service, and more". White paper, SOA World magazine, Vol. 4(6).

[18] Ran.S. "A Model for Web Services Discovery with QoS”,2004, ACM SIGEcom, Vol. 4(1), pp.1-10.

[19] Liu, Y., Ngu, A. and Zheng, L., 2004, "QoS Computation and Policing in Dynamic Web Service Selection", Proceedings of WWW 2004

[20] Eyhab Al- Masri, and Qusay H. Mahmoud, 2007, "QoSbased Discovery and Ranking of Web services", Proceedings of IEEE International Conference.

[21] M.Adel Serhani, Rachida Dssouli, Abdelhakim Hafid, Houari Sahraoui, " A QoS broker based architecture for efficient web services selection", Proceedings of the IEEE International Conference on Web Services (ICWS'05), 